\title{
COMMUNITY-BASED ELECTROPHYSIOLOGICAL ABNORMALITIES IN CHILDREN WITH ADHD: TransLating RESEARCH FINDINGS INTO A Clinical SETTING
}

\author{
Cynthia N. Martin ${ }^{1 *} \&$ Lukasz M. Konopka $a^{1,2,3}$ \\ ${ }^{1}$ Chicago School of Professional Psychology, Chicago, Illinois, USA \\ ${ }^{2}$ Advanced Clinical Neuroscience, Wheeling, Illinois , USA \\ ${ }^{3}$ Loyola University Medical Center Department of Psychiatry, Maywood, Illinois, USA
}

\begin{abstract}
Attention Deficit Hyperactivity Disorder (ADHD) is a highly prevalent disorder of childhood that is behaviorally characterized by repeated episodes of inattention, impulsivity, and hyperactivity. Selecting appropriate treatment based on presenting behavior can be challenging and usually requires timeintensive medication trials. Clinical and research communities agree there is a need for an objective method to both evaluate symptoms of ADHD and determine appropriate treatment options (Barkley, 2009). We are proposing the use of qEEGs (quantitative electroencephalograms) in patients presenting with ADHD symptomology to provide an objective assessment of underlying neuropathology that can aid in diagnostic clarification, thus offering targeted treatment approaches. Current studies have identified three abnormal electrophysiological clusters (Chabot \& Serfontein, 1996; Clarke, et al., 2001; Monastra, et al., 2001). This study was designed to examine these clusters of ADHD in a naturalistic sample to determine congruity between current research literature and frontline community treatment centers.

We evaluated 30 pediatric patients $(6-17 \mathrm{yrs})$ in a naturalistic psychiatric setting. As described in Konopka (2005), participants completed an initial baseline study to examine brain electrophysiology through qEEG. Electrophysiological clusters were defined by (Chabot \& Serfontein, 1996; Clarke, et al., 2001; Monastra, et al., 2001): cortical hypoarousal (excess theta), cortical hyperarousal (excess beta, decreased theta/delta), and maturation lagged (excess delta/theta). Neuroguide software and databases (Thatcher, 2009) were used for spectral analyses to convert raw EEG data to quantifiable form (qEEG). Fast Fourier Transformation was used to generate relative power maps for comparison of electrophysiological abnormalities. Comparisons were made for cortical activity levels of Delta, Theta, Alpha, and Beta. The database was used to statistically compare ADHD children to a control group $(\mathrm{N}=678)$, normed for gender, age, and handedness.

Results show that children with ADHD exhibited abnormal electrophysiological activity in comparison to age and gender matched norms. While we found the previously documented electrophysiological groups, there was a disproportionately high degree of cortically hyperaroused children within the sample. Specifically, the overwhelming majority of our sample presented with excess relative beta in the central-parietal regions. Additionally we identified a novel electrophysiological group, which displayed concurrent excesses of fast (beta) and slow (theta) activity in relative frequencies.

These findings illustrate discrepancies in ADHD populations studied in clinical centers and research laboratories. Given that there is agreement for the need of objective assessment tools and that imaging modalities provide promise (Chabot, Orgill, Craw ford, Harris, \& Serfontein, 1999), there is also a need
\end{abstract}

*Correspondence to: Cynthia N. Martin, e-mail: cnm9319@ego.thechicagoschool.edu

Received December 2, 2011; accepted December 15, 2011; Act Nerv Super (Praha) 53(3-4),129-40. 
for continuity between research and clinical communities. This study provides an important objective diagnostic and treatment paradigm that can be highly informative for the direction of future studies.

Key w ords: ADHD; EEG; Fast Fourier Transformation; hyperarousal; hypoarousal

\section{INTRODUCTION}

\subsection{Community-based electrophysiological abnormalities in children with ADHD: Translating research findings into a clinical setting}

Attention-deficit hyperactivity disorder (ADHD) is one of the most common childhood psychiatric illnesses today. Currently, as many as fifty percent of children whom are seen by psychiatrists receive a diagnosis of ADHD (Halperin, Marks, \& Schulz, 2008). Current frontline treatment of ADHD in pediatric patients involves medications that actively alter brain chemistry. Medications are used, despite widely agreed upon neurobiological standards for diversities in the ADHD diagnosis (Barkley, 2010; Loo \& Barkley, 2005; McGough \& Barkley, 2004). Current diagnostic and treatment assessment methods rely on clinical interview, observer report, and cognitive testing, which attempt to infer neurobiological diversity based on behavior.

Currently, diagnostic criteria are based on symptom clusters that, in children, are difficult to differentiate from typical development and other diagnoses (Rims, 2011; Gupta \& Kar, 2010; Morley, 2010). Behavioral symptoms similar to ADHD cannot easily be teased apart from differential diagnoses through observation and self-report measures. For instance, impulsivity is a hallmark symptom of ADHD, however this symptom is also highly prevalent in other childhood disorders, such as pediatric bipolar. While the observable behavioral symptom of impulsivity between the two diagnostic groups will look quite similar, the underlying neuropathology is inherently different (Passarotti, Sweeney, \& Pavuluri, 2010). Thus given the importance in understanding the relation between a child's behavioral symptoms with underlying brain functioning, clinical and research communities agree there is a need for additional objective methods for evaluating the neurobiological presentation of patients with ADHD symptoms (Barkley, 2009; Chabot \& Serfontein, 1996; Clarke, et al., 2001a; Konopka \& Poprawski, 2008; Lubar, 1985; Monastra, et al., 2001; Posner \& Petersen, 1990; Rubia, et al., 1999; Sow ell, et al., 2003).

We are proposing the use of quantitative electroencephalography (qEEG) in patients presenting with ADHD symptomology to provide an objective assessment of underlying neuropathology. Electroencephalography (EEG) studies have consistently [for the last 75 years (Jasper, 1935)] identified differences between typically developing children and those who display symptom clusters of ADHD. Over the last 30 years, with advanced technological resources, the use of qEEG has allowed researchers to further differentiate electrophysiological subgroups of children who present with similar behavioral symptoms of ADHD (Chabot \& Serfontein, 1996; Clarke, et al., 2001a; Konopka \& Poprawski, 2008; Lubar, 1985; Monastra, et al., 2001; Posner \& Petersen, 1990; Rubia, et al., 1999; Sow ell, et al., 2003).

Similarly, EEG studies have found variability in the diagnostic etiology of ADHD that can have tremendous effects on responsiveness to treatment (Clarke, et al., 2001a). Although stimulant medications are currently the front line treatment for attention disorders, $30-40 \%$ of pediatric patients fail to respond, or negatively respond to psychotropic medication (Halperin, Marks, \& Schulz, 2008). Understanding differences in electrophysiology of the brain, leading to categorizing pediatric ADHD from a brain-to-behavior perspective, as compared to behavior to biology, can aid clinicians in having a better understanding of symptom etiology thus offering more targeted and efficacious treatment options (Clark, et al., 2005). To date, three subclusters of electrophysiological abnormalities have been consistently identified and include: hypoaroused cortical profile, maturational lag, and 
hyperaroused profile (Chabot \& Serfontein, 1996; Clarke, et al., 2001a; Clarke, Barry, McCarthy, \& Selikowitz, 2001b; Monastra, et al., 2001).

\subsection{Hypoaroused Electrophysiological Profile}

Excess slow wave activity with dominant frequencies in the theta band has classically defined ADHD samples (Satterfield et al., 1972, Mann et al., 1992, Janzen et al., 1995, Chabot \& Serfontein, 1996, Lazzaro et al., 1998, Clark et al., 1998, 2001b,c). Excess relative theta activity during eyes closed resting state has defined 42 to $68 \%$ of the ADHD samples in current research studies (Clarke, et al., 2001a; Clarke, Barry, McCarthy, Selikowitz, \& Brown, 2002). This profile, in addition to excess theta activity, is generally associated with reduced beta activity and normal resting state alpha activity. As first noted in 1932 (Jasper, 1932) excess theta activity is positively associated with cortical normalization secondary to psychostimulant administration. These studies have been replicated across the last 30 years and continue to support positive therapeutic response through normalization of excess cortical slowing (Clark et al., 2001; Chabot \& Serfontein, 1996, Lazzaro et al., 1998; Janzen et al., 1995).

\subsection{Maturational Lag Cortical Profile}

This profile is associated with increased slow wave activity in both the delta and theta bands, with a concurrent reduction in fast wave activity, primarily in the beta band (Satterfield et al., 1972, Mann et al., 1992, Janzen et al., 1995, Clark et al., 1998, 2001b,c). This profile has similar features to the hypoaroused cortical profile. Prominent deficits can be noted in the posterior regions of the brain when examining relative delta and alpha, and central regions in relative beta (Chabot \& Serfontein, 1996, Lazzaro et al., 1998). The topography of th is profile would be typical of electrophysiological activity present in a younger child. The dominant frequency in early childhood is delta; therefore children that show excessive levels of delta activity may be maturationally behind their peers rather than on an independent developmental course. This profile has higher variability in terms of pharmacological treatment responsiveness. Generally, maturationally lagged children have higher rates of EEG normalization following administration of dexamphetamine and have poor treatment responses when administered methylphenid ates (Clarke, Barry, McCarthy, Selikowitz, 2002).

\subsection{Hyperaroused Electrophysiological Profile}

Excess beta activity and concurrent deficiencies in theta, delta, and/ or alpha activity characterize the hyperaroused profile. While this profile has been reported in several studies (Chabot \& Serfontein, 1996; Clark et al., 1998, 2001c,d,e) it is less prevalent than the previously mentioned EEG profiles that are characterized by excess slow wave activity. Hyperaroused electrophysiological profiles typically present in $0-20 \%$ of recent research samples (Chabot \& Serfontein, 1996; Clark et al., 1998, 2001c,d,e; Snyder \& Hall, 2006). Pharmacological treatment with this population is varied and generally associated with negative responsiveness to methylphenid ates and dexamphetamine (Clark et al., 1998; 2001; Snyder \& Hall, 2006).

\subsection{Hypothesis}

Given that current studies are based solely on hypothesis driven, recruitment based patient populations; this questions the applicability of these data to clinical practice. The 
translational aspect of these data is important to address as the applicability of these EEG patterns in frontline treatment centers may vary. This study serves to examine the prevalence and utility of electrophysiological cluster patterns of pediatric ADHD that have been identified in current research studies. Efforts are made to determine if there is congruity between the research based and community based naturalistic samples. To date, this is the first study to examine electrophysiological trends of ADHD in children who present for the sole purpose of clinical treatment in a community outpatient center.

\section{METHODS}

We evaluated 30 patients (5-17 yrs) in a naturalistic community based outpatient psychiatric setting (see table 1 for demographic information). All patients were seeking treatment due to parent referral. Patients received standard outpatient care and neurodiagnostic work up. Study data was extracted from archival patient data between January 2009 and August 2009. Patients were predominately right handed, and further the sample was well balanced between genders with nineteen males, and eleven females (see table 1). Each patient received electrophysiological (qEEG) and behavioral assessments that are a routine procedure, in the clinic prior to treatment. Inclusion criteria included negative history for developmental and/ or acquired brain injury, seizure disorder, and no comorbid major mood or conduct disorders. This led to the exclusion of 8 children in the final analysis. All patients were medication free at the time of the EEG acquisition.

Table 1: Total Sample Demographics

\begin{tabular}{ll}
\hline & \\
\hline Age & $\begin{array}{l}\text { Mean 11 } \\
\text { (7-16 years) }\end{array}$ \\
$\begin{array}{l}\text { Gender } \\
\quad \text { Male }\end{array}$ & 19 \\
$\quad$ Female & 11 \\
Handedness & \\
$\quad$ Left & 3 \\
$\quad$ Right & 27 \\
Diagnosis & \\
$\quad$ ADHDin \\
(inattentive type) \\
$\quad \begin{array}{l}\text { ADHDcom } \\
\text { (combination type) }\end{array}$
\end{tabular}

Note. 30 patients' electrophysiological assessments were analyzed for the purpose of this study. The majority of the sample was diagnosed with ADHD combined type with ages ranging from 7-16 years old.

As described in Konopka (2005), participants completed an initial baseline study to examine brain electrophysiology through qEEG. Diagnostic status was agreed on by two separate medical professionals; all patients met DSM-IV-TR criteria for ADHDcom (combined type) or ADHDin (primarily inattentive type) according to a combination of two or more of the following: Structured clinical interview, parent report, self/ parent/ teacher 
report measures, performance on a continuous performance test, and clinical observations. As such, 26 children met criteria for ADHDcom, and four children met criteria for ADHDin. No significant trends in EEG findings differed across behavioral diagnosis (see table 2).

The patient's baseline brain activity was assessed via electroencephalography (EEG). Data acquisition was done by a registered EEG technician and acquired in an eyes-closed, resting state. Nineteen cephalic electrodes were individually applied to the patient's scalp by a qualified EEG technician. Placement of the electrodes was based on the international 10/20 system. Impedance for each electrode was $<5.0 \mathrm{KOhms}$ and was monitored throughout the data acquisition to ensure the stability of the recording. Ear lobes were used as active leads and the tip of the nose was used as the reference point. The EEG data was acquired with Syn-Amp 32 Channel amplifier system by Neuroscan. The sampling rate was at $500 \mathrm{~Hz}$ per channel. The filter settings were $0.01 \mathrm{~Hz}$ and $70 \mathrm{~Hz}$. Non-cephalic electrodes were used to monitor eye movement and EKG. The standard protocol also involved basic activation procedures such as hyperventilation, photic stimulation, and twenty minutes of sleep; however these data were not analyzed for the purpose of this study. The patient was in a dedicated quiet room for the assessment.

A board certified ECNS clinician (LMK) selected artifact free epochs during eyes closed states that excluded drowsiness and then converted these epochs into their frequency domains using Fast Fourier Transform. Individual data were compared to a normative database through NeuroGuide V1.5 commercial software.

Topographical maps were generated to reflect comparison of the patient to a normative database. The NeuroGuide V1.5 database contains 678 normal controls that are matched for age, gender, and handedness (Thatcher, 1998). Topographical maps displaying z-score deviations in relative frequencies for Delta, Theta, Alpha, and Beta were used. Two blind, independent raters sorted the topographical maps based on the deviation maps. Patient's without EEG findings were excluded from further analyses, which included two patients. Agreement between raters exceeded .95. Categories were established based on classically defined qEEG patterns (hypoaroused, maturational lag, and hyperaroused) in relative frequencies. Inclusion criteria included abnormalities $\geq 2$ standard deviations from the means established through the normative database. Topographical maps that did not align with established categories were grouped based on the unique topography of the maps.

Table 2: Electrophysiological Band Subcluster.

\begin{tabular}{|c|c|c|c|}
\hline & $\begin{array}{l}\text { Number } \\
\text { of Patients }\end{array}$ & Gender & Diagnosis \\
\hline Hypoaroused & 4 & $\begin{array}{l}\text { Male } 4 \\
\text { Female } 0\end{array}$ & $\begin{array}{l}\text { ADHDin } 1 \\
\text { ADHDcom } 3\end{array}$ \\
\hline Maturational Lag & 3 & $\begin{array}{l}\text { Male } 2 \\
\text { Female } 1\end{array}$ & $\begin{array}{l}\text { ADHDin } 0 \\
\text { ADHDcom } 3\end{array}$ \\
\hline Hyperaroused & 6 & $\begin{array}{l}\text { Male } 4 \\
\text { Female } 1\end{array}$ & $\begin{array}{l}\text { ADHDin } 0 \\
\text { ADHDcom } 5\end{array}$ \\
\hline Combination & 6 & $\begin{array}{l}\text { Male } 4 \\
\text { Female } 2\end{array}$ & $\begin{array}{l}\text { ADHDin } 1 \\
\text { ADHDcom } 5\end{array}$ \\
\hline Delta Deficit & 1 & $\begin{array}{l}\text { Male } \\
\text { Female } 1\end{array}$ & $\begin{array}{l}\text { ADHDin } \\
\text { ADHDcom } 1\end{array}$ \\
\hline Normal & 2 & $\begin{array}{l}\text { Male } 2 \\
\text { Female } 0\end{array}$ & $\begin{array}{l}\text { ADHDin } 1 \\
\text { ADHDcom } 1\end{array}$ \\
\hline
\end{tabular}

Note. Six EEG patterns were identified. No gender differences were noted as male and female patients were spread across groups. Eight patients were excluded due to history of neurological (seizure) or developmental disorder, comorbid major mood or psychotic episode. Inclusion diagnosis included ADHD primarily inattentive type (ADHDin) and ADHD combined type (ADHDcom). 
Based on rater selection, five groups were identified (see table 2): (1) Cortically hypoaroused $(\mathrm{N}=4)$ : Excess relative theta, reduced relative delta and relative beta activity; (2) Maturational lag $(\mathrm{N}=3)$ : Excess relative delta and relative theta activity with reduced relative beta; (3) Hyperaroused $(\mathrm{N}=6)$ : Excess relative beta, and reduced relative theta and/ or delta; (4) Combination Group $(\mathrm{N}=6)$ : Combination of excess high relative alpha/ beta with concurrent excess in relative delta/ theta activity; (5) Delta Deficit Group ( $\mathrm{N}=1$ ): Deficits in relative delta activity, with no concurrent excess high relative alpha/ beta.

Following categorization based on individual normative findings, group analyses were conducted for the most prominent EEG cluster findings. This included the Hypoaroused (excess theta, $\mathrm{N}=4$ ), Hyperaroused (excess beta, $\mathrm{N}=6$ ), and Combination (excess theta and beta, $\mathrm{N}=6$ ) groups. Independent t-test between groups was conducted to compare peak differences and topography. Further, global findings in relative power were analyzed through one-way ANOVA. Global findings are defined as the sum percentage of all 19electrodes in relative power frequency. Tukey HSD post hoc comparison tests were subsequently used to analyze between group differences.

\section{RESULTS}

Twenty children displayed EEG patterns in relative power that significantly deviated in comparison to the normative database. Analyses show clear between group differences within the ADHD sample (see figure 1). The hypoaroused group accounts for $13.33 \%$ of the total sample $(\mathrm{N}=4)$. This group shows significantly higher levels of abnormal theta activity at $\mathrm{Cz}$ lead $(\mathrm{p}<.01)$ as compared to the Hyperaroused group. Interestingly, the Hyperaroused group $(\mathrm{N}=6)$ accounts for a larger portion of the sample at $20 \%$, and as compared to Hypoaroused group shows clear differences in abnormal parietal-central beta $(\mathrm{p}<.01)$. Novel findings include the Combination group $(\mathrm{N}=6)$, which accounts for another $20 \%$ of the ADHD sample. This novel group has not yet been identified in the research literature and shows abnormal patterns of excess parietal-central beta $(\mathrm{p}<.01)$ and theta at $\mathrm{Cz}$ lead $(\mathrm{p}<.01)$ as compared to Hypoaroused and Hyperaroused groups, respectively.

\section{Relative Percentage Findings}

One-way ANOVA show significant differences for relative theta percentages across 19electrodes for the three identified subclusters $[F(2,54)=33.24, p>.0001]$. Similarly, there were significant differences for relative beta across 19 -electrodes for the three subclusters also found $[\mathrm{F}(2,54)=24.68, \mathrm{p}>.0001]$. Tukey HSD post hoc comparison tests were further used to analyze between group differences (see figure 2). Findings are indicated below.

Hypoaroused group compared to Hyperaroused group: Post hoc comparisons using the Tukey HSD test indicated that the Hypoaroused group $(M=25.58, \mathrm{SD}=6.14)$ demonstrates significant differences $(p>.001)$ in global relative theta activity as compared to the Hyperaroused group $(M=12.10, S D=3.55)$. Conversely, within beta frequencies, the Hyperaroused group $(M=20.95$, $\mathrm{SD}=4.81)$ show s significant global differences $(\mathrm{p}<.01)$ as compared to the Hypoaroused group $(\mathrm{M}=11.57, \mathrm{SD}=4.03)$.

Hypoaroused group compared to Combination group: Post hoc comparisons using the Tukey HSD test indicated that the Hypoaroused group $(M=25.58, S D=6.14)$ demonstrates significant differences $(\mathrm{p}>.001)$ in global relative theta activity as compared to the Combination group $(M=20.60, S D=5.1)$. Conversely, within beta frequencies, the Combination group $(M=20.60$, $\mathrm{SD}=5.10)$ show significant global differences $(\mathrm{p}<.01)$ as compared to the Hypoaroused group $(\mathrm{M}=11.57, \mathrm{SD}=4.03)$.

Combination group compared to Hyperaroused group: Post hoc comparisons using the Tukey HSD test indicated that the Combination group $(M=20.6, S D=5.1)$ demonstrates significant differences $(p>.001)$ in global relative theta activity as compared to the Hyperaroused group $(\mathrm{M}=12.1 .58, \mathrm{SD}=3.55)$. However, there were no significant differences found between the 
Combination $(\mathrm{M}=20.60, \mathrm{SD}=5.1)$ and Hyperaroused $(20.95, \mathrm{SD}=4.81)$ groups when looking at beta frequency, supporting that the global abnormal elevations are of similar abnormality.

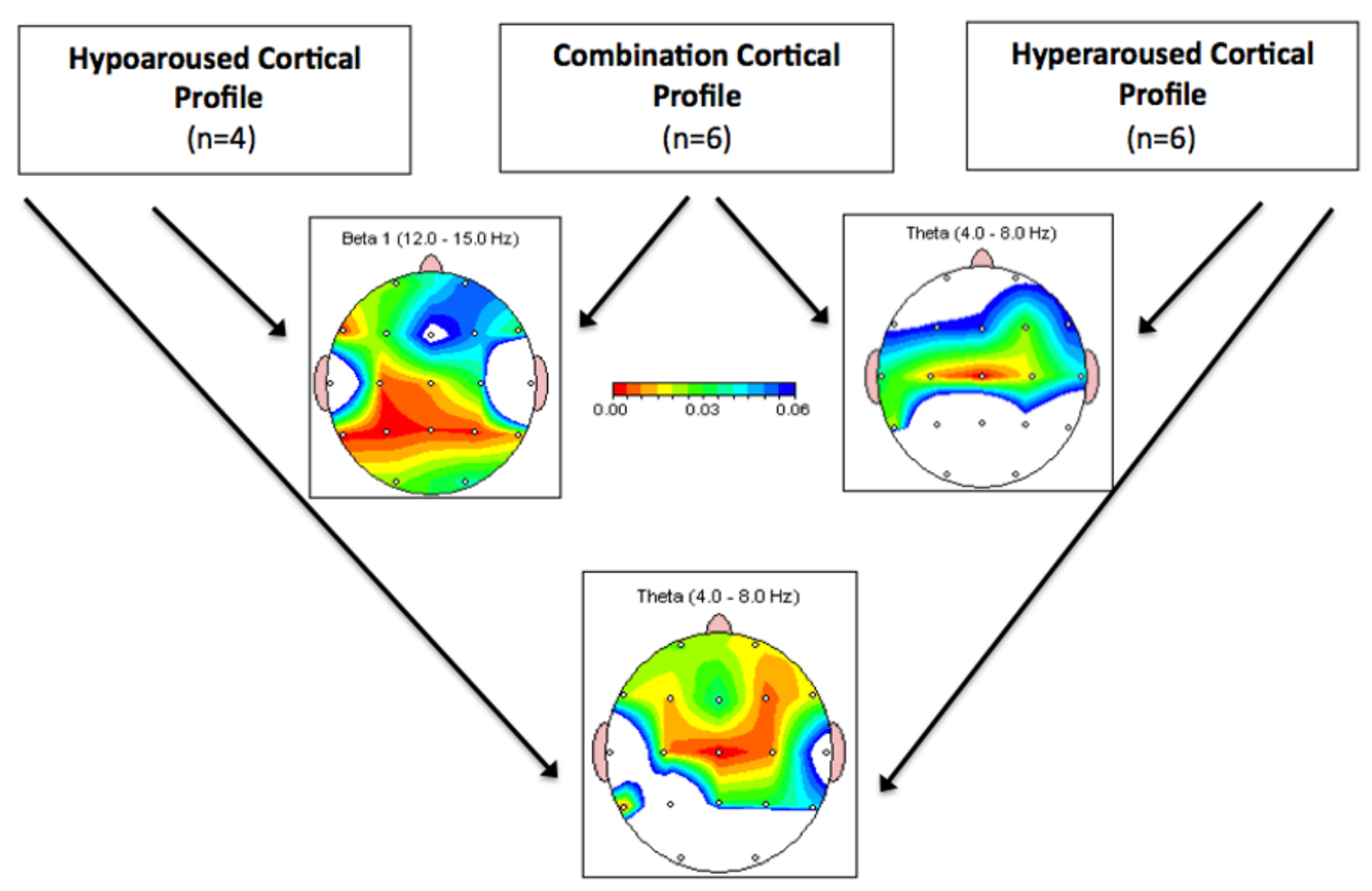

Figure 1. Topographical localization differences in relative power.

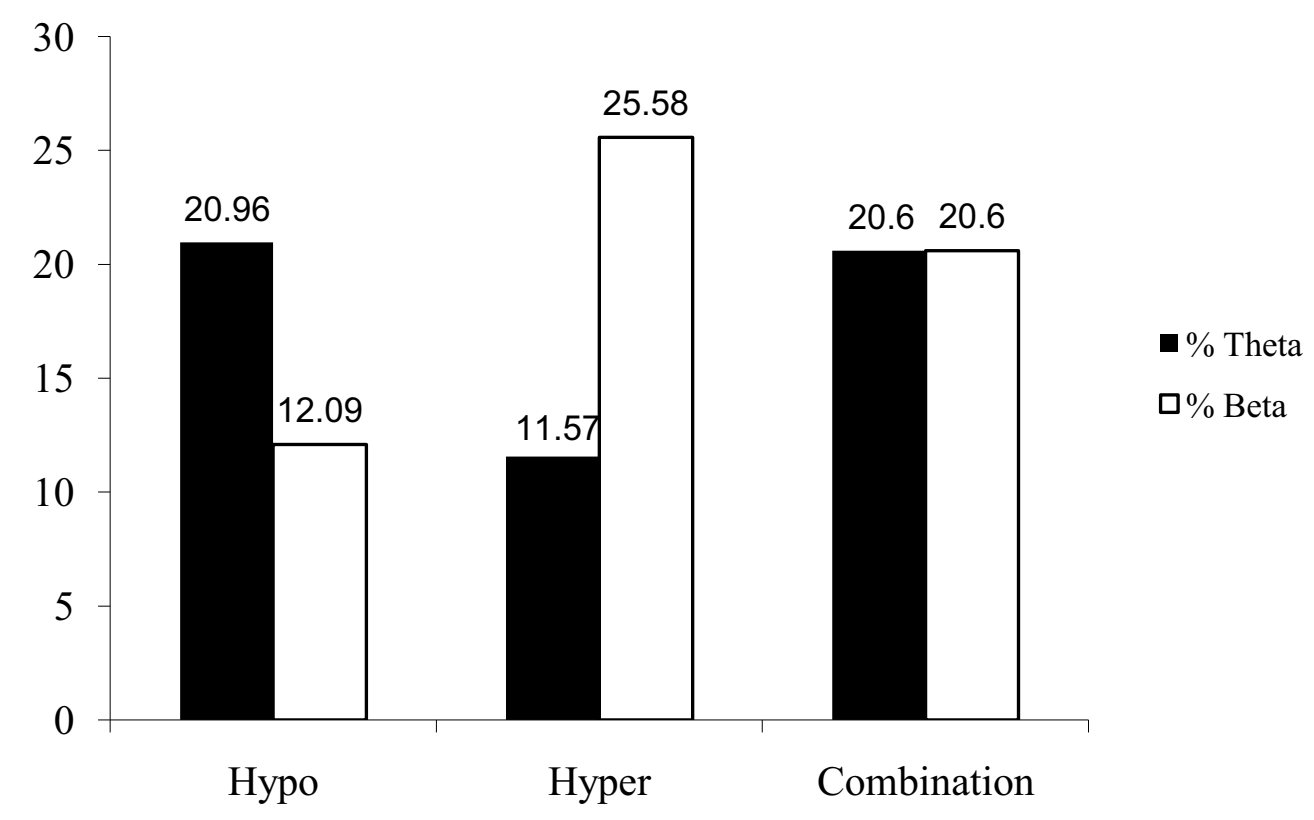

Figure 2. Relative percentage differences between group. 
Note Fig. 1. Significant differences between groups are indicated. Hypoaroused group shows significantly higher levels of abnormal theta activity at $\mathrm{Cz}$ lead $(\mathrm{p}<.01)$ as compared to the Hyperaroused group. Combination group shows concurrent higher levels of parietal-central abnormal beta as compared to the Hypoaroused group $(\mathrm{p}<.01)$ and higher abnormal theta at $\mathrm{Cz}$ lead as compared to Hyperaroused group $(\mathrm{p}<.01)$. Beta 1 findings are defined as beta frequencies between 12 and $15 \mathrm{~Hz}$. For the purpose of this study, Beta findings over $15.5 \mathrm{~Hz}$ were not analyzed.

Note Fig. 2. Significant between group differences in relative power are indicated. Tukey's HSD post hoc analyses show clear theta and beta patterns across groups. The Hypoaroused group displayed excess global relative theta activity as compared to the Hyperaroused group. The Hyperaroused group demonstrated excess global relative percentage of Beta activity as compared to the Hypoaroused group. The Combination group demonstrated significantly elevated relative levels of theta and beta activity concurrently.

\section{DISCUSSION}

Essential findings of this study were as follows. First, our findings replicate current literature trends in that ADHD samples do not represent a homogeneous group and that EEG is a valid and efficacious tool in identifying these subpopulations. Children diagnosed with ADHD were consistently found to display abnormal electrophysiological patterns as compared to age and gender controlled norms. Of the 30 children evaluated, 28 displayed abnormal EEG findings within relative power frequencies. Further, as consistent with previous studies (Satterfield et al., 1972, Mann et al., 1992, Janzen et al., 1995, Clark et al., 1998, 2001b,c), within our ADHD sample we identified the hypoaroused, maturationally lagged, and hyperaroused qEEG patterns. However, the prevalence rates of classical EEG cluster presentations (i.e., hypoaroused and maturationally lagged) represented the lowest portion of our sample with concurrent high rates of the atypical EEG subcluster (hyperaroused group). This finding highlights an important finding in the potential discrepancy between recruitment style and naturalistic sampling patterns and will be further discussed below.

Moreover, we show for the first time a unique electrophysiological cluster that presents in children with an ADHD diagnosis. This abnormal profile displayed a concurrent combination of increases in fast and slow wave activity respectively. Termed the "combination group" this pattern was characterized by elevations in relative parietal beta frequencies $(12-15 \mathrm{hz})$ with simultaneous elevations in central theta (4-8hz). Secondly, our clinical sample displayed high rates of atypical EEG patterns, specifically within relative beta frequency. This was seen across two subclusters including the hyperaroused and combination groups. These groups showed elevated beta frequencies (12-15hz) and represented over half of our clinical sample. Previous studies suggest that increased beta activity in an ADHD sample is relatively rare, representing $0-13 \%$ of study populations to date.

We also found that cortical slowing, which was most prominent in the hypoaroused group, accounted for a relatively small portion of this naturalistic sample. This is highly discrepant with previous studies (Clarke et al., 2001; Monestra, 1998; Chabot, 2005), which largely finds the hypoaroused cortical profile to represent a greater portion of the ADHD samples, independent of behavioral label. The majority of children in our clinical sample were exhibiting excess relative beta in their baseline EEG. Given that this electrophysiological profile is correlated with higher symptom severity, emotional reactivity (Clark et al., 2001e), and higher rates of negative pharmacological treatment responsiveness (Loo, Hop fer, Teale, \& Reite, 2004; Clarke, Barry, McCarthy, Selikowitz, Clarke, \& Croft; 2003) the finding sheds further light on a seemingly large discrepancy between naturalistic and recruitment based research samples. 


\section{CONCLUSIONS}

Novel findings of this study are particularly important because they shed light on the probable notion that clinical samples have increased heterogeneity among children with an ADHD diagnosis. This can have significant implications on treatment responsiveness. As previously discussed, children displaying excess beta in their resting state EEG have an increased likelihood of not responding, or negatively responding to traditional treatment with stimulant medications (Clark et al., 1998; 2001; Snyder \& Hall, 2006). Limited studies have shown normalization of beta activity following pharmacological treatment and thus highlight the need for further research.

As our findings were largely comprised of atypical EEG patterns, and suggest that increased cortical slowing is actually the least representative of children seeking treatment at an outpatient, community center we offer several considerations that may account for the sampling bias. First, estimates of prevalence rates of ADHD severity between treatment and research facilities are greatly varied in that inclusion and exclusion criteria are not universal (Polanczyk, Lima, Horta, Biederman, Rohde, 2007). Previous studies have found that children diagnosed with ADHD who have dominant frequencies in the beta band typically present with a higher intensity of ADHD symptoms, moodiness, and temper tantrums (Clark et al., 2001e). Not surprising, given that that these children are more disruptive, they are likely more commonly referred for immediate clinical treatment rather than recruited for research studies, which could account for some of variability found in our sample. This notion is further supported by current behavioral research that suggests there is a bias towards more disruptive expressions of ADHD in clinical samples (Willcutt, 2010; Willcutt \& Carlson, 2005).

In summary, these findings suggest that while current EEG research is translatable to clinical settings there is increased variability in clinical populations and increased portions of children exhibiting atypical ADHD (i.e. hyperaroused and combination) cortical patterns. This can have significant implications on the individual patient's responsiveness to treatment. Pharmacological treatment with this population is varied, as children with excessive beta activity in their resting state EEG are generally demonstrating negative responsiveness to methylphenidates and dexamphetamines (Clarke, et al., 2001). Interestingly, children with hypoaroused cortical profiles demonstrate the highest rates of responsiveness to psychostimulant treatment but may present in outpatient clinics at lower rates as evident by our sample proportions.

\subsection{Study Limitations}

Given the nature of our study, several limitations were inevitable. Naturalistic samples inherently lack the internal validity compared to recruitment style samples. While this was closely considered and strict clinic procedures are followed, many of the children's data extracted for the purpose of this study did not have consistent behavioral measures (i.e. continuous performance task) that could be readily correlated with subcluster findings. Further, while we sought to include all children with an ADHD diagnosis inclusion criterion placed limitations on our sample size. In addition, despite a behavioral presentation of ADHD symptoms and meeting full criteria for the disorder, two children extracted for the purpose of our study were found to have normal EEG patterns as compared to the normative database and thus could not be included in the final analyses. This led to limitations in that our final sample size was relatively low $(\mathrm{N}=20)$. Thus these findings will require replication from a larger naturalistic sample. Given the limited time frame sample data was extracted from it is recommended that obtaining larger samples across multiple treatment centers would be beneficial. 


\subsection{Directions for future research}

Our findings provide additional support and evidence for shifting the paradigm in ADHD research and treatment. Children with ADHD continue to be grouped based on behavioral symptoms that do not take into account differing and overlapping neurobiological substrates of the disorder. Specifically, there is substantial evidence that speaks to the heterogeneity with pediatric ADHD, suggesting that as clinicians it is imperative that we understand the child's individual electrophysiology if we are going to use an active neuromodulator that alters brain chemistry. This study highlights the discrepancy in EEG presentation between recruitment style and naturalistic samples, with an increased prevalence in atypical EEG patterns. Thus we suggest that further research be conducted to investigate hyperaroused and combination electrophysiological profiles. Understanding the behavioral correlates to these presentations as well as the drivers of treatment responsiveness will increase the efficacy of diagnostic and treatment options for this population.

As such, we highly stress the importance for a paradigm shift that utilizing neurobiological subtyping as a way to define study populations. Given the increasing evidence supporting the neurobiological heterogeneity of ADHD samples, subtyping can be a useful method to clearly differentiate and define ADHD groups. While subtyping based on EEG profile has been deemed efficacious, more recently this model has been adapted within imaging research streams that utilize complementary functional imaging paradigms, such as fMRI. A recent study by Durston, Belle, and Zeeuw, (2011) show that while ADH D is largely a disorder of the prefrontal cortex that may have similar behavioral symptoms of impulsivity and inattention, there are variable subcortical pathways affected in children. They too stress the importance of sub grouping children based on these variations in subcortical-cortical pathways as a way to offer better diagnostic clarification to aid in more targeted, individualized, and efficacious treatment options. Thus, given the preponderance of evidence that similar behavioral symptoms do not correlate with homogenous neurobiological findings, we recommend that further studies categorize ADHD subtypes based on brain-tobehavior findings to enhance both research methodology and clinical practice.

\section{FUNDING DISCLOSURE}

This study was supported in part by the Faculty Grant to LMK from TCSPP.

\section{REFERENCES}

Almeida, L. G., Ricardo-Garcell, J., Prado, H., Barajas, L. z., Fern`ndez-Bouzas, A., ¡vila, D., et al. Reduced right frontal cortical thickness in children, adolescents and adults with ADHD and its correlation to clinical variables: A cross-sectional study. Journal of Psychiatric Research, 44(16), 12141223.

Association, A. P. (2000). DSM-IV Diagnostic and statistical manual of mental disorders (4 $4^{\text {th }} \quad$ ed.). Washington DC American Psychiatric Press

Barkley, R. A. (1990). A critique of current diagnostic criteria for attention deficit hyperactivity disorder: Clinical and research implications. Journal of Developmental and Behavioral Pediatrics, 11(6), 343-352. doi:10.1097/ 00004703-199012000-00014

Barkley, R. A. (2003). Child Psychopathology (Vol. 2 ). New York: Guilford Press.

Loo, Hopfer, Teale, \& Reite, (2004) EEG Correlates of Methylphenidate Response in ADHD: Association With Cognitive and Behavioral Measures. Journal of Clinical Neurophysiology 21 (6), 457-464.

Loo, S. K., \& Barkley, R. A. (2005). Clinical Utility of EEG in Attention Deficit Hyperactivity Disorder. Applied Neuropsychology, 12(2), 64-76. doi:10.1207/ s15324826an1202_2

Barkley, R. A. (2009). Commentary on Hyper-activity in children having behavior disorders (Childers, 1935). Journal of Attention Disorders, 13(3), 229-231. doi: 13/3/ 229 [pii] 10.1177/ 1087054709338857 
Barkley, R. A. (2010). Against the status quo: Revising the diagnostic criteria for ADHD. Journal of the American Academy of Child \& Adolescent Psychiatry, 49(3), 205-207. doi:10.1097/ 00004583-20100300000002

Barry, R. J., Clarke, A. R., \& Johnstone, S. J. (2003). A review of electrophysiology in attentiondeficit/hyperactivity disorder: I. Qualitative and quantitative electroencephalography. Clinical Neurophysiology, 114(2), 171-183.

Castellanos, F. X. (2001). Neural substrates of attention-deficit hyperactivity disorder. Advances in Neurology, 85, 197-206.

Castellanos, F. X., Giedd, J. N., Marsh, W. L., Hamburger, S. D., Vaituzis, A. C., Dickstein, D. P., et al. (1996). Quantitative brain magnetic resonance imaging in attention-deficit hyperactivity disorder. Archives of General Psychiatry, 53(7), 607-616.

Castellanos, F. X., Lee, P. P., Sharp, W., Jeffries, N. O., Greenstein, D. K., Clasen, L. S., et al. (2002). Developmental trajectories of brain volume abnormalities in children and adolescents with attention-deficit/ hyperactivity disorder. Journal of American Medical Association, 288(14), 1740-1748. doi: joc20194 [pii]

Chabot, R. J., di Michele, F., \& Prichep, L. (2005). The role of quantitative electroencephalography in child and adolescent psychiatric disorders. Child and Adolescent Psychiatric Clinics of North America, 14(1), 21-53.

Chabot, R. J., Orgill, A. A., Crawford, G., Harris, M. J., \& Serfontein, G. (1999). Behavioral and electrophysiologic predictors of treatment response to stimulants in children with attention disorders. Journal of Child Neurology, 14(6), 343-351.

Chabot, R. J., \& Serfontein, G. (1996). Quantitative electroencephalographic profiles of children with attention deficit disorder. Biological Psychiatry, 40(10), 951-963.

Clarke, A. R., Barry, R. J., McCarthy, R., \& Selikowitz, M. (2001a). EEG-defined subtypes of children with attention-deficit/ hyperactivity disorder. Clinical Neurophysiology, 112(11), 2098-2105.

Clarke, A. R., Barry, R. J., McCarthy, R., \& Selikowitz, M. (2001b). Excess beta activity in children with attention-deficit/ hyperactivity disorder: An atypical electrophysiological group. Psychiatry Research, 103(2), 205-218.

Clarke, A. R., Barry, R. J., McCarthy, R., Selikowitz, M., \& Brown, C. R. (2002). EEG evidence for a new conceptualisation of attention deficit hyperactivity disorder. Clinical Neurophysiology, 113(7), 10361044.

Clarke, A. R., Barry, R. J., McCarthy, R., Selikowitz, M, Clarke, D.C., \& Croft, R.J. (2003) Effects of stimulant medications on children with attention-deficit/ hyperactivity disorder and excessive beta activity in their EEG. Clinical Neurophysiology, 114(9), 1729-1737.

Conners, C. K. (2004). Validation of Adhd Rating Scales. Journal of American Academy of Child \& Adolescent Psychiatry, 43(10), 1190-1191.

Cubillo, A., Halari, R., Ecker, C., Giampietro, V., Taylor, E., \& Rubia, K. Redu ced activation and interregional functional connectivity of fronto-striatal networks in adults with childhood AttentionDeficit Hyperactivity Disorder (ADHD) and persisting symptoms during tasks of motor inhibition and cognitive switching. Journal of Psychiatric Research, 44(10), 629-639.

Durston, S., Hulshoff Pol, H. E., Schnack, H. G., Buitelaar, J. K., Steenhuis, M. P., Minderaa, R. B., et al. (2004). Magnetic resonance imaging of boys with attention-deficit/ hyperactivity disorder and their unaffected siblings. Journal of A merican A cademy of Child and Adolescent Psychiatry, 43(3), 332-340. doi: 00004583-200403000-00016 [pii]

Durston, S., \& Konrad, K. (2007). Integrating genetic, psychopharmacological and neuroimaging studies: A converging methods approach to understanding the neurobiology of ADHD. Developmental Review, 27(3), 374-395.

Filipek, P. A., Semrud-Clikeman, M., Steingard, R. J., Renshaw, P. F., Kennedy, D. N., \& Biederman, J. (1997). Volumetric MRI analysis comparing subjects having attention-deficit hyperactivity disorder with normal controls. Neurology, 48(3), 589-601.

Gupta, R., \& Kar, B. R. (2010). Specific cognitive deficits in ADHD: A diagnostic concern in differential diagnosis. Journal of Child and Family Studies, 19(6), 778-786. doi:10.1007/ s10826-010-9369-4

Hill, D. E., Yeo, R. A., Campbell, R. A., Hart, B., Vigil, J., \& Brooks, W. (2003). Magnetic resonance imaging correlates of attention-deficit/ hyperactivity disorder in children. Neuropsychology, 17(3), 496-506.

Hoffman, D. A., Lubar, J. F., Thatcher, R. W., Sterman, M. B., Rosenfeld, P. J., Striefel, S., et al. (1999). Limitations of the American Academy of Neurology and American Clinical Neurophysiology Society paper on QEEG. Journal of Neuropsychiatry and Clinical Neuroscience, 11(3), 401-407. 
Hynd, G. W., Semrud-Clikeman, M., Lorys, A. R., Novey, E. S., \& Eliopulos, D. (1990). Brain morphology in developmental dyslexia and attention deficit disorder/hyperactivity. Archives of Neurology, 47(8), 919-926.

Jensen, P. S., Kettle, L., Roper, M. T., Sloan, M. T., Dulcan, M. K., Hoven, C., et al. (1999). Are stimulants overprescribed? Treatment of ADHD in four U.S. communities. Journal of A merican A cademy of Child and Adolescent Psychiatry, 38(7), 797-804.

Kates, W. R., Frederikse, M., Mostofsky, S. H., Folley, B. S., Cooper, K., Mazur-Hopkins, P., et al. (2002). MRI parcellation of the frontal lobe in boys with attention deficit hyperactivity disorder or Tourette syndrome. Psychiatry Research, 116(1-2), 63-81. doi: S0925492702000665 [pii]

Konopka, L. M., \& Poprawski, T. J. (2008). qEEG Studies of Attention Disorders and Mood Disorders in Children In A. Ivanenko (Ed.), Sleep and Psychiatric Disorders in Children and Adolescents Informa Healthcare.

Lubar, J. F. (1985). EEG biofeed back and learning disabilities. Theory Into Practice, 24(2), 106-111.

Monastra, V. J., Lubar, J. F., \& Linden, M. (2001). The development of a quantitative electroencephalographic scanning process for attention deficit,Äihyperactivity disorder: Reliability and validity studies. Neuropsychology, 15(1), 136-144.

Morley, C. P. (2010). Disparities in ADHD assessment, diagnosis, and treatment. International Journal of Psychiatry in Medicine, 40(4), 383-389. doi:10.2190/ PM.40.4.b

Mostofsky, S. H., Cooper, K. L., Kates, W. R., Denckla, M. B., \& Kaufmann, W. E. (2002). Smaller prefrontal and premotor volumes in boys with attention-deficit/ hyperactivity disorder. Biological Psychiatry, 52(8), 785-794. doi: S0006322302014129 [pii]

McGough, J. J., \& Barkley, R. A. (2004). Diagnostic controversies in adult attention deficit hyperactivity disorder. The American Journal of Psychiatry, 161(11), 1948-1956.

Passarotti, AM, Sweeney, A., Pavuluri MN. Neural Correlates of Response Inhibition Deficits in Pediatric Bipolar Disorder and Attention Deficit Hyperactivity Disorder (2010). Psychiatry Research: Neuroimaging. Psychiatry Research, 181(1);36-43.

Posner, M. I., \& Petersen, S. E. (1990). The attention system of the human brain. Annual Review Neuroscience, 13, 25-42. doi: 10.1146/ annurev.ne.13.030190.000325

Polanczyk G, de Lima MS, Horta BL, Biederman J, Rohde LA. (2007) The worldwide prevalence of ADHD: a systematic review and metaregression analysis (2007). American Journal of Psychiatry. 164(6):942-8.

Raz, S., Debastos, A. K., Newman, J. B., \& Batton, D. Extreme prematurity and neuropsychological outcome in the preschool years. Journal of the International Neuropsychological Society, 16(1), 169-179.

Rimm, S. (2011). Attention deficit/ hyperactivity disorder: A difficult diagnosis. In J. L. Jolly, D. J. Treffinger, T. Inman, J. Smutny, J. L. Jolly, D. J. Treffinger, ... J. Smutny (Eds.), Parenting gifted children: The authoritative guide from the National Association for Gifted Children (pp. 399-404). Waco, TX US: Prufrock Press. Retrieved from EBSCOhost.

Rubia, K., Overmeyer, S., Taylor, E., Brammer, M., Williams, S. C., Simmons, A., et al. (1999). Hypofrontality in attention deficit hyperactivity disorder during higher-order motor control: a study with functional MRI. American Journal of Psychiatry, 156(6), 891-896.

Shaw, P., Eckstrand, K., Sharp, W., Blumenthal, J., Lerch, J. P., Greenstein, D., et al. (2007). Attention deficit/ hyperactivity disorder is characterized by a delay in cortical maturation. Proceedings of the National A cademy of Science USA, 104(49), 19649-19654.

Sow ell, E. R., Thompson, P. M., Welcome, S. E., Henkenius, A. L., Toga, A. W., \& Peterson, B. S. (2003). Cortical abnormalities in children and adolescents with attention-deficit hyperactivity disorder. Lancet, 362(9397), 1699-1707.

Thatcher, R. W., Lubar, Joel, F. (2009). History of the scientific standards of QEEG normative databases In A. Press (Ed.), Introduction to EEG and Neurofeedback (Second ed., pp. 29-59). San Diego: Academic Press 